Artigo de Revisão

\title{
Análise da coerência do espectro do eletrencefalograma
}

\author{
EEG spectral coherence
}

Renato Anghinah ${ }^{1}$

\section{RESUMO}

Apresentamos uma revisão sobre a análise da coerência espectral do EEG, abordando desde a compreensão de sua definição até a aplicabilidade em pesquisa e as perspectivas de utilização futura na prática clínica, com enfoque especial para distúrbios cognitivos e os quadros demenciais.

Unitermos: Eletrencefalograma quantitativo, Análise de coerência, Demência.

Citação: Anghinah R. Análise da coerência do espectro do eletrencefalograma. Rev Neurociencias 2005; 13(1):050-053.

\section{SUMMARY}

We showed in this review about EEG spectral coherence how you understand its concept and applicability in research and future perspectives in clinical practice uses, with main focus in cognitive disturbs and dementia.

\section{Keywords: Quantitative electroencephalography, Coherence analysis, Dementia}

Citation: Anghinah R. EEG spectral coherence. Rev Neurociencias 2005; 13(1):050-053.

\section{INTRODUÇÃO}

O EEG quantitativo (EEGq) é um exame topográfico - funcional, distinto da tomografia computadorizada e da ressonância nuclear magnética, que são exames de imagem morfológica ou estrutural, sendo sua indicação distinta destes ${ }^{1}$. Por este motivo, a análise quantitativa e topográfica tem como base o traçado do EEG, sendo apenas uma evolução tecnológica deste, sem substituí-lo².
Dois conceitos fundamentais na análise quantitativa de séries temporais, em particular do EEG, são os conceitos de análises no "domínio do tempo" e no "domínio da freqüência". Quando se considera como variável independente o tempo, como em um evento bem caracterizado que ocorre em um determinado instante do tempo (por exemplo: um paroxismo por ponta-onda aos dois minutos de registro de um exame de EEG), o sinal é considerado no "domínio do tempo", ou seja, na própria escala Trabalho realizado: Grupo de Neurologia Cognitiva e do Comportamento, Departamento de Neurologia e Setor de EEG de alta Resolução do LIM-27 IPq,
Faculdade de Medicina da Universidade de São Paulo.

1 - Doutor em neurologia pela FMUSP, médico do grupo de neurologia cognitiva e do comportamento da FMUSP, responsável pelo setor de Eletroencefalografia Clínica do Lab. de Neurociências (LIM27) IPq FMUSP. 
em que o sinal é representado por um grafoelemento ou uma freqüência versus a amplitude ou a potência deste sinal|3-9.

A análise no domínio da frequência vale-se de um importante teorema matemático, o teorema de Fourier, que garante que qualquer sinal periódico pode ser decomposto em um conjunto de funções senóides e cossenóides, denominadas bases ortogonais, de várias freqüências múltiplas da freqüência fundamental, que da mesma forma, em operação reversa, somando-se todos os seus componentes resulta no sinal original ${ }^{4}$.

Por exemplo, um traçado com atividade alfa (que a olho nu aparenta ser uma seqüência de ondas na freqüência alfa) podem ser compostas por outras freqüências como as atividades beta, teta ou delta e ainda harmônicas e sub-harmônicas das mesmas de menor amplitude ${ }^{5}$ e ao serem sobrepostas, resultam em uma atividade elétrica com aparência a olho nu de atividade alfa. A transformação rápida de Fourier, "Fourier fast transform" (FFT), é uma implementação algorítmico - computacional muito eficiente utilizada para se decompor os sinais de EEG nas suas diferentes freqüências ${ }^{4}$.

No "domínio da freqüência" o estudo de um evento no tempo, como uma espícula ou uma variante da normalidade, não será possível, pois na decomposição no domínio de freqüência perde-se a informação de tempo, ou melhor, a relação temporal dos eventos paroxísticos pela decomposição dos eventos transitórios do EEG em dois componentes, freqüência e fase $\mathrm{f}^{4}$. Pode - se apresentar os resultados das análises obtidos com a quantificação do sinal do EEG de várias maneiras, tais como, histogramas, gráfico de linhas ou de barras, tabelas ou de forma cartográfica9 .

\section{Análise de Coerência}

Coerência espectral quadrática, coerência ordinária ou simplesmente coerência é a medida da covariância da potência espectral, dentro de bandas de freqüência específicas, entre dois canais (normalmente determinada a partir da estimativa do espectro cruzado) do EEG. Estudos em pacientes com agenesia de corpo caloso mostram uma diminuição da amplitude da coerência interhemisférica em ampla banda de freqüência indicando que a medida de coerência seria um indicador funcional da relação entre os hemisférios cerebrais via comissural ${ }^{10-11}$.

Matemática e computacionalmente obtém-se a coerência entre dois canais dividindo - se a estimativa da potência do espectro cruzado destes dois canais pelos auto - espectros de cada um dos canais ${ }^{3,10-13}$ (Equação 1). Em um traçado com 20 canais de registro, é possível obter 190 (um dos 20 eletrodos $\times 19$ eletrodos restantes, dividido por dois) combinações de medidas individuais de coerência inter-eletrodos, por cada banda de freqüência. Simplificando este processo, os programas comerciais de análise computacional mostram a coerência apenas entre os pares de canais (eletrodo ativo com referência inativa; exemplo F7 - Referência auricular) homólogos dos dois hemisférios cerebrais para os eletrodos do sistema 10-20, excluindo-se os eletrodos de linha média e auriculares, resultando em oito eletrodos por hemisfério cerebral (FP1FP2; F7-F8; F3-F4; C3-C4; T3-T4; P3-P4; T5-T6; 01-02) com 16 medidas de coerência interhemisféricas, para cada faixa de freqüência. Alguns programas de análise de EEG permitem uma livre relação de medida entre os eletrodos, mensurando também coerências intra-hemisféricas, (dentro do mesmo hemisfério cerebral) ${ }^{3,10-12}$.

$$
C^{2}(f)=\frac{G_{x y}^{2}(f)}{G_{x x}(f) G_{y y}(f)}
$$

Equação 1. Coerência entre par de canais

em que $G_{x y}^{2}(f)$ é a densidade de potência do espectro cruzado e $G_{x x}(f)$ e $G_{y y}(f)$ são as densidades espectrais dos sinais $x$ e $y$.

\section{Estudos das redes Neurais e Demência}

Coerência espectral ou simplesmente coerência é a medida da covariância do espectro de dois sinais do EEG. A alta coerência entre dois sinais do 
EEG tem sido considerada como uma evidência da possível existência de uma conexão funcional entre duas áreas corticais captadas pelo registro do EEG ${ }^{14}$. Coerência é, portanto, uma medida de co-ativação de duas ou mais áreas cerebrais. A conectividade estrutural não pode ser determinada pela coerência espectral ordinária uma vez que um par de estruturas, sem uma conexão direta entre si, pode ter alta coerência ao ser simultaneamente ativada por uma terceira estrutura.

Desde os primeiros estudos sobre a análise quantitativa do $E \mathrm{EG}^{15-17}$ propiciou determinar o espectro de potência e outros parâmetros estatísticos do EEG com sua ampla utilização. A informação obtida da função de coerência parece ser essencial para o entendimento de como a inter-relação funcional entre regiões pode mudar sob diferentes condições ou ao longo de um comportamento ${ }^{18}$.

Dentro do grande número de possibilidades de correlações entre diferentes áreas cerebrais, a análise de coerência tem sido comprovada como sendo capaz de expor as relações inter - hemisféricas mediadas pelo corpo caloso, tanto na vigília como no sono ${ }^{13}$, sendo explorada em vários protocolos clínicos no estudo das demências. Leuchter et al. ${ }^{18}$ encontraram uma coerência menor em pacientes com DV em relação a pacientes com DA, porém não evidenciaram diferença estatisticamente significante entre o grupo DA e o grupo controle formado por indivíduos normais; Besthorn et al. ${ }^{19}$ estudaram um grupo de 50 pacientes com hipótese diagnóstica de DA e compararam-no a um grupo controle normal, encontrando uma coerência diminuída nas regiões centrais e frontais, achado este semelhante ao relatado por outros estudos ${ }^{13,20}$.

As diferenças encontradas no comportamento da coerência das bandas lentas (delta e teta) e das bandas rápidas (alfa e beta) do EEG provavelmente aparecem em momentos distintos da evolução da DA e, portanto, devem refletir substratos neuropatológicos específicos para esta etapa da doença. A diminuição da coerência das bandas rápidas ocorre nas fases iniciais da DA e reflete uma perda das redes neurais córtico corticais, enquanto o aumento da coerência para bandas lentas ocorre em fases mais avançadas e estaria associada à deaferentação das estruturas subcorticais ${ }^{10}$.
Vários estudos mostram que há uma diminuição na coerência inter-hemisférica nas regiões posteriores de pacientes com diagnóstico provável de DA quando comparados a grupos controles normais ${ }^{21-23}$. Achado este também corroborado por Anghinah et al. ${ }^{24}$ que estudaram a coerência inter-hemisférica do espectro do EEG, obtido pela FFT, da região occipital do escalpo (eletrodos 01 e O2) para a banda de freqüência alfa $\left(\right.$ alfa $_{1} ; 8,0$ a $10,0 \mathrm{~Hz}$, e alfa $; 10,5$ a 12,5 Hz) em indivíduos normais maiores de 50 anos e pacientes com diagnóstico provável de DA. Neste estudo foi encontrada uma diminuição na coerência inter-hemisférica dos indivíduos com DA para ambas as sub-bandas do ritmo alfa $^{24}$.

Portanto, até o presente, a análise de coerência do EEG tem sido bem sucedida em diferenciar grupos de indivíduos com DA da população normal porém, a sua especificidade entre diferentes formas de demência e alta variabilidade inter-individual ainda torna esta metodologia questionáve ${ }^{25}$.

Comparando-se a sensibilidade da análise de coerência com outros métodos diagnósticos das demências, observa-se forte correlação entre as alterações da coerência e testes neuro-psicológi$\cos ^{26-27}$, SPECT ${ }^{28}$ e PET ${ }^{29}$. A combinação de vários métodos funcionais, como SPECT e EEGq aumentaria a sensibilidade e especificidade diagnósticas ${ }^{29}$. Outro estudo conclui que a atividade teta do EEG relaciona-se com os achados do PET (metabolismo da glicose) para a região temporal e que a atividade alfa do EEG teria forte correlação funcional com o metabolismo da glicose na região occipital ${ }^{30}$.

Em recente estudo comparamos dois grupos etários, adultos jovens com idosos saudáveis com idade acima de 65 anos, e obtivemos resultados estatisticamente significativos de que não há diferenças entre as médias de coerência interhemisférica na banda de freqüência alfa entre os indivíduos adultos nas duas faixas etárias estudados, portanto a diminuição da coerência para a banda alfa, poderia ser indicativa de alguma alteração funcional, por vezes detectada de modo antecipado a manifestação clínica ${ }^{31}$, porém estudos adicionais, com casuística mais ampla, são necessários para comprovação desses achados. 


\section{REFERÊNCIAS BIBLIOGRÁFICAS}

1. Orrison WW, Lewine JD, Sanders JA, Hartshorne MF. Functional brain imaging. St Louis: Mosby 1995:327-368.

2. Lesser RP. Guidelines Committees of American Electroencephalographic Society. J Clin Neurophysiol 1994;11: 09-11.

3. Gotman J, Gloor P, Ray WF. A quantitative comparison of traditional reading of the EEG and interpretation of computer-extracted features in patients with supra tentorial brain lesions. Electroencephalogr Clin Neurophysiol 1975; 38:623-639.

4. Gotman J. The use of computers in analysis and dysplay of EEG and evoked potencials. In: DD Daly, AT Pedley (ed). Current Practice of Clinical Electroencephalography, 2nd ed. New York: Raven Press 1990; 51-84.

5. Wong PKH. Introduction to brain topography. New York: Plenum Press 1991;150-162.

6. Epstein CM. Digital EEG: Choosing the right equipment for your lab. In: 30th Annual course in clinical EEG and Electrophisiology of American EEG Society. Washington, DC American EEG Society/Executive office-PO BOX 30 Bloomfield, CT 06002-USA, 1995

7. Luccas FJC, Braga NIO, Fonseca LC, Frochtengarten ML. Recomendações para o registro e interpretação do mapeamento topográfico do eletrencefalograma (EEG) e potenciais evocados sensoriais (PES) parte I: aspectos gerais. Braz J Epilepsy Clin Neurophysiol 1996;2:175-182.

8. Nuwer MR. Quantitative EEG analysis in clinical settings. Brain Topogr 1996:8:201-208.

9. Nuwer MR, Comi G, Emerson R, et al. IFCN standards for digital recording of clinical EEG. Electroencephalogr Clin Neurophysiol 1998;106:259-261.

10. Kuks JBM, Vos JE, O'Brien MJ. Coherence patterns of the infant sleep EEG in absence of the corpus callosum. Electroencephalogr clin neurophysiol 1987;66:8-14

11. Nielsen T, Montplaisir J, Lassonde M. Decreased Interhemisferic EEG Coherene during sleep in agenesis of the Corpus Calosum. Eur Neurol 1993;33:173-176.

12. Tucker DM, Roth DL, Bait TB. Funcional conections among cortical regions: Topography of EEG coherence. Electroenceph Clin Neurophysiol 1986;63:242-250.

13. Locatelli T, M Cursi, D Liberati, M Franceschi, G Comi. EEG coherence in Alzheimer's disease. Electroencephalogr Clin Neurophysiol 1998;106:229-237.

14. Harmony T, Marosi E, Fernandez T, Bernal J, Rodriguez M, Reyes A. EEG coherences in patients with brain lesions. Intern J Neuroscience 1994;74:203-226

15. Lehmann D. Multichannel topography of human alpha EEG fields. Electroenceph Clin Neurophysiol. 1971, 31: 439-449.

16. Duffy FH, Burchfiel JL, Lombroso CT. Brain electrical activity mapping (BEAM): A method for extending the clinical utility of EEG and evoked potential data. Ann Neurol 1979;5:309-321.

17. Duffy FH, lyer VG, Surwillo WW. Clinical electroencephalogra- phy and topographic brain mapping. New York: Springer Verlag 1989;99-132.

18. Leuchter AF, Spar JE, Walter DO, Weiner H. Electroen-cephalographic spectra and coherence in the diagnosis of Alzheimer's-type and multi-infarct dementia. Arch Gen Psychiatr 1987;44:993-998

19. Besthorn C, Förstl H, Geiger-Kabisch C, Sattel H, Gasser T. EEG coherence in Alzheimer disease. Electroencephalogr Clin Neurophysiol 1994;90:242-245

20. Anderer P, Saletu B, Klöppel B, Semlitsch HV, Werner H. Discrimination between demented patients and normals based on topographic EEGslow wave activity: comparison between z statistics, discriminant analysis and artificial neural network classifiers. Electroencephalogr Clin Neurophysiol 1994;91:108-117

21. Rosen I. EEG as diagnostic tool in dementia. Dement Geriatr Cogn Disord 1997;8:110-116

22. Stevens A, Kircher T. Cognitive decline unlike normal aging is associated with alterations of EEG temporo-spatial characteristics. Eur Arch Psychiatr Clin Neurosci 1998;248:259-292.

23. Wada Y, Nanbu Y, Koshino Y, Yamaguchi N, Hashimoto T. Reduced interhemispheric EEG coherence in Alzheimer disease: analysis during rest and photic stimulation.Alzheimer. Dis Assoc Disord 1998;12:175-181

24. Anghinah R, Kanda PAM, Jorge MS, LimaEEP, Pascuzzi L, Mello ACP. Estudo da Coerência do eletroencefalograma para a banda de frequência alfa em indivíduos adultos normais e com provável demência do tipo Alzheimer. Arq Neuropsiquiatr 2000;58(2A):272-275

25. Leocani L, Comi G. EEG coherence in pathological conditions. J Clin Neurophysiol 1999;16(6):548-555

26. Dunkin JJ, Osato S, Leuchter AF. Relationships between EEG coherence and neuropsychological tests in dementia. Clin Electroencephalogr 1995;26:47-59

27. Sarnthein J, Petsche H, Rappelsberger P, Shaw GL, Stein A. Synchronization between prefrontal and posterior association cortex during human working memory. Neurobiol 1998:95:7092-7096.

28. Sloan EP, Fenton GW, Kennedy NS, Maclennan JM. Neurophysiology and spect cerebral blood fow patterns in dementia. Electroencephalogr Clin Neurophysiol 1994;91:163-170.

29. Szelies B, Mielke R, Herholz K, Heiss WD. QEEG compared to FDG PET for classification of vascular and degenerative dementia. Electroencephalogr Clin Neurophysiol 1994;91(2):131-139.

30. Szelies B, Mielke R, Kessler J, Heiss WD. EEG power changes are related to regional cerebral glucose metabolism in vascular dementia. J Neurol Scienc 1999;110 (4):615-620

31. Anghinah R, Caramelli P, Takahashi DY, Nitrini R, Sameshima K. EEG alpha band coherence analysis in healthy adults: preliminary results. Arq Neuropsiquiatr 2005;63(1):83-86. 